\title{
Effectiveness of an offer of the Smoke Free smartphone application for smoking cessation: protocol for a randomised controlled trial
}

\author{
Sarah E. Jackson ${ }^{1}$, Olga Perski ${ }^{1}$, David Crane ${ }^{1}$, Susan Michie ${ }^{2}$, Robert West ${ }^{1}$, Jamie Brown ${ }^{1,2}$ \\ ${ }^{1}$ Department of Behavioural Science and Health, University College London, UK \\ ${ }^{2}$ Department of Clinical, Educational and Health Psychology, University College London, UK
}

Corresponding author: Sarah E Jackson, PhD. Department of Behavioural Science and Health, University College London, 1-19 Torrington Place, London WC1E 6BT

Tel: (44) 2076793179

Fax: (44) 2079168354

s.e.jackson@ucl.ac.uk

Running head: Effectiveness of a smoking cessation app

Word count: 4,020

Declarations of interest: DC is the originator of the Smoke Free app and derives income from it. RW has undertaken research and consultancy for and receives travel funds and hospitality from manufacturers of smoking cessation medications (Pfizer, GlaxoSmithKline and Johnson and Johnson). JB has received unrestricted research funding from Pfizer, who manufacture smoking cessation medications. OP, RW and JB are unpaid members of the scientific steering group of the Smoke Free mobile application.

Key words: randomised controlled trial; smartphone app; smoking cessation; intervention; cessation aid. 


\section{Abstract}

Background and aims: Smartphone applications (apps) hold promise for delivering tobacco smoking cessation support to large numbers of people at low unit cost. Smoke Free is an evidence-informed, widelyused app that is highly rated by users. This study aims to assess its effectiveness compared with no support.

Design: Two-arm individually randomised controlled effectiveness trial.

Setting: Online trial with no restrictions on location.

Participants: English-speaking smokers aged $\geq 18$ years willing to make a quit attempt within 4 weeks from initial contact $(n=4,990)$.

Measurements: The primary outcome measure is self-reported 26-week continuous smoking abstinence. Secondary outcome measures include quit attempts in the first 4 weeks post-randomisation, 12-week continuous smoking abstinence, and 26-week continuous smoking abstinence among those who made a quit attempt.

Comments: If it is effective, the Smoke Free smartphone app is an affordable and widely implementable intervention to help smokers to quit. 


\section{Introduction}

Tobacco smoking remains one of the leading causes of preventable death worldwide (1). The majority of smokers want to quit $(2)$ and many try to quit each year $(3,4)$, but the chances of success of any given quit attempt are low, with fewer than $5 \%$ of unaided attempts succeeding for 12 months or more (5). Smartphone applications (apps) may aid quit attempts at very low unit cost. This study aims to assess the effectiveness of a widely used smoking cessation app compared with no support.

A wide range of smoking cessation interventions are known to increase the prevalence and success of quit attempts (6). Because of the widespread ownership of smartphones, apps hold promise for delivering smoking cessation support to large numbers of people at low unit cost in a format that is readily accessible at almost any time (7-9). Around two-thirds of the world's population now owns a smartphone (10) and in 2017, there were 178 billion app downloads (11). At present, there are no documented health risks associated with the use of smoking cessation apps. Preliminary evidence suggests that delivering smoking cessation interventions via apps may improve abstinence rates compared with attempting to stop smoking unaided (12-20). In two prospective studies of smokers using cessation apps $(16,18)$ and three randomised controlled trials (RCTs) that compared an app with a text messaging intervention or less comprehensive app (13-15), self-reported success rates were higher than would be expected from unaided cessation. In another RCT, an app acting as a decision aid for smokers interested in quitting resulted in higher 6-month abstinence rates than an information-only app (20). These studies have been limited by relatively small samples (the majority $n<350$ ). No published RCTs have compared apps designed to provide ongoing support with unaided quitting.

With over 4 million downloads to date and 130,000 new users each month, Smoke Free (smokefreeapp.com) is one of the world's most widely used smoking cessation apps. The app is evidenceinformed, available for iOS and Android OS and has a mailing list of $>170,000$ users who have opted to accept further contact from the app. In a large exploratory RCT, the full version of the app increased 3month self-reported complete abstinence rates compared with a reduced version (21). It therefore constitutes a useful test bed for assessing the effectiveness of a smartphone app for smoking cessation.

Recruiting participants for an evaluation of smoking cessation apps can be challenging. One possible source is past users of an app. It can be assumed that the large majority of past users on the app's mailing list will have resumed smoking even if the app improves cessation rates above what is achieved by unaided 
cessation, so this provided a potential source of recruits for the present trial. How useful such a recruitment source will be depends on uptake of the offer of repeated use of the app as well as the effectiveness of the app for smokers who have used it previously. There is reason to believe that both uptake rates and effectiveness will be sufficient for this study. In terms of uptake, research has found that smokers have a high likelihood of re-trying a method of quitting they used previously (22). In terms of effectiveness, a large RCT of a range of smoking cessation interventions found that previous use of a given intervention did not significantly reduce its effectiveness in the current attempt (23). The Smoke Free mailing list will not be the only source of recruitment but it is hoped that it will provide a large percentage of the required sample. With all data collected remotely with no need for face-to-face interaction, evaluation of the app can be conducted without restrictions on location, producing generalisable estimates of effectiveness.

This study aims to assess the effectiveness of the Smoke Free app in increasing rates of tobacco smoking cessation compared with follow-up only. Given that, in an effectiveness trial such as this, not all those offered the intervention will take it up, the test is of the offer rather than actual use of the app; how far the results reflect actual app effectiveness will depend on the proportion of participants taking up the offer. Thus we will address the following primary research question (RQ) and endpoint:

1. In English-speaking adult smokers willing to quit in the next 4 weeks who are recruited online or have previously used the Smoke Free app and agreed to be followed up [population] and in an unrestricted setting [setting], how effective is an offer to use the app plus follow up [intervention] compared with no offer of the app and follow-up only [comparator] in promoting self-reported smoking cessation for at least 26 weeks, assessed 30 weeks after enrolment [outcome 1]?

We will also address the following secondary RQs and endpoints:

2. In the population, setting and intervention versus comparator for RQ1, what is the effect in promoting at least one quit attempt in the 4 weeks following enrolment in the study [outcome 2]?

3. In the population, setting and intervention versus comparator for RQ1, what is the effect in promoting smoking cessation for at least 12 weeks, assessed 4 weeks after enrolment [outcome 3]?

4. In the setting, intervention versus comparator and outcome for RQ1, what is the effect in those who make at least one quit attempt in the 4 weeks following enrolment in the study [population 2]?

5. In the population, setting and intervention versus comparator for RQ1, what is the effect in promoting downloading or using the Smoke Free app at least once, assessed 30 weeks after enrolment [outcome 4]? 
6. Are the answers to RQs 1 to 5 different in smokers who are: a) male versus female [population moderator 1], b) more versus less addicted to cigarettes [population moderator 2], c) 18-35 versus $\geq 35$ years of age [population moderator 3], d) with versus without post-16 educational qualifications [population moderator 4], e) from poorer versus richer financial situations [population moderator 5], f) have previous versus no prior experience with the app [population moderator 6]?

\section{Method}

\section{Study design and participants}

This will be a two-arm individually randomised controlled effectiveness trial. Participants will be adult smokers who are intending to quit in the next 4 weeks, willing to participate in a 30-week study of their smoking and able to provide consent. Table 1 describes the eligibility criteria.

[Table 1 here]

Individuals who are not eligible for participation in the trial will be informed of this but provided with a link to the Smoke Free app and information about the best ways of stopping smoking.

\section{Sample size}

We aim to recruit 4,990 participants; 2,495 in each group. The intended sample size is based on achieving $90 \%$ power to detect a risk ratio (RR) of at least 1.5 with an alpha of $p<0.052$-tailed in response to RQ1. The RCT of the full Smoke Free app versus a reduced version (21) found a RR of 1.9 (95\% Cl 1.5-2.4), risk difference of $0.7 \%$, with missing equals smoking analysis and a follow-up rate of $8.5 \%$. The expected followup rate in the current study is $>50 \%$. The expected effect size is less than the previous RCT because not all of those allocated to the intervention condition will use the app.

\section{Recruitment}

A key strategy for recruitment will be to identify potential participants from a mailing list of $>170,000$ smokers who have previously signed up to the Smoke Free app and have agreed to be contacted in the future. We will approach individuals who signed up to the app $>6$ months previously 1,000 at a time via 
email with an invitation to participate in the study. After each wave, the numbers recruited will be assessed to determine whether changes need to be made to the recruitment procedures. The target recruitment rate will be 50 per 1,000 contacted.

In order to improve the generalisability of the sample, and in case the response rate from the mailing list is lower than we anticipate, we will also use a variety of other recruitment channels, including social media and paid online advertising (e.g. Facebook, Twitter).

Smokers will be informed that the study involves completing an initial online questionnaire (baseline questionnaire) and further online questionnaires 4, 16 and 26 weeks later (follow-up questionnaires), and that those interested in quitting may be randomly offered support. They will signal interest and consent to participate in the study by clicking on a link to the baseline questionnaire.

\section{Compensation}

Potential participants will be informed at baseline that on completion of all the follow-up assessments a donation of $£ 10$ or equivalent in local currency will be made on their behalf to Cancer Research UK or Macmillan Cancer Support, according to their preference.

\section{Randomisation}

Those who are eligible and consent to participate in the trial will be randomised at that point to intervention or comparator conditions. Randomisation will be 1:1 at the individual level with no restriction (i.e. no blocking) and will be automated using a random number function.

\section{Blinding}

The investigators will be blinded to participants' treatment allocation until all data have been collected.

\section{Interventions}

\section{Comparator}

After consenting, participants in the comparator condition will receive a message encouraging them to make a quit attempt within the next 4 weeks and reminding them of the importance of responding to follow-up requests which are designed to track their progress. 
Intervention

Participants in the intervention condition will receive the same advice as those in the comparator condition plus offer of the full version of the Smoke Free app free of charge, encouragement to use the app, and a link to download it.

The Smoke Free app is based on behaviour change techniques that would be expected from theory (21) and evidence with face-to-face support $(24,25)$ to aid smoking cessation. It guides smokers through the first month of their quit attempt by helping them maintain their resolve by setting a clear goal, monitoring their progress towards that goal and becoming aware of benefits of being smoke-free achieved to date. The app has several components: 1) a calculator which tracks the total amount of money not spent on buying cigarettes and the number of cigarettes not smoked ('Dashboard'); 2) a calendar which tracks the amount of time elapsed since cessation ('Dashboard'); 3) a scoreboard which awards virtual 'badges' to users for not smoking ('Badges'); 4) progress indicators which inform users of the health improvements made since the start of their quit attempt (e.g. pulse rate, oxygen levels, carbon monoxide levels; 'Dashboard') ; 5) a diary which tracks the frequency, strength, location and triggers of cravings to smoke ('Diary'); 6) a graph which displays the frequency, location, strength and triggers of cravings to smoke ('Cravings'); 7) daily missions which are assigned from the start of a user's quit date for one calendar month ('Missions') and 8) a chatbot which delivers evidence-based guidance about quitting smoking via a conversational interface which resembles text messaging. See Table 2 for individual behaviour change techniques included in the Smoke Free app, coded against a 44-item taxonomy of behaviour change techniques used in individual behavioural support for smoking cessation (26).

[Table 2 here]

\section{Outcomes}

The primary outcome will be the percentage of participants reporting not having smoked for 26 weeks at the 30-week follow-up. On the basis of the intention-to-treat principle, those who do not respond to followup attempts will be retained in the analyses and classified as continuing smokers (27). Secondary outcomes will be the percentage of participants reporting (i) quit attempts at 4-week follow-up, defined as having made a serious quit attempt in the last 4 weeks; (ii) smoking cessation for at least 12 weeks at the 16-week follow-up; (iii) smoking cessation for at least 26 weeks at the 30-week follow-up in those who made a quit 
attempt; and (iv) app use, defined as downloading or using the Smoke Free app at least once at any point during the study period.

\section{Procedures}

Participants will be assessed at 0 (baseline), 4, 16 and 30 weeks after enrolment. Table 3 summarises the schedule of enrolment and follow-up assessments for trial participants.

[Table 3 here]

Potential participants will engage with the study via recruitment emails sent to individuals on the Smoke Free mailing list or in response to online advertising. They will be given information about the study and those who consent will access the baseline questionnaire via an online link. Those who meet the eligibility criteria, assessed via the questionnaire, will be automatically randomised to the intervention or comparator condition.

All questionnaires will be conducted online via Qualtrics (Qualtrics, Provo, UT; https://www.qualtrics.com). The baseline questionnaire will assess the following: email address, mobile phone number (optional), smartphone ownership, motivation to quit in the next month (Motivation To Stop Scale (28)), willingness to complete online questionnaires after 4, 16 and 30 weeks, age (18-34, 35-64, $\geq 65$ years), sex (male, female), education (any versus no post-16 qualifications), financial status (live comfortably, meet needs with a little left, just meet basic expenses, don't meet basic expenses) (29), country of residence, first language (English, other), number of cigarettes smoked per day, cigarette addiction (first cigarette within 5 minutes, 6-30 minutes, 31-60 minutes, >60 minutes of waking), history of serious quit attempts (never, yes - not in the past year, yes - in the past year), and past and current use of support for smoking cessation (prescription nicotine replacement therapy (NRT), NRT bought over the counter, varenicline, bupropion, face-to-face behavioural support, telephone support, written self-help materials, websites, apps).

In an exploratory addition to outcome assessment, participants who have a heart rate monitoring device (e.g. a FitBit, Apple watch) will be asked to report their average resting heart rate as indicated by their app. Heart rate declines substantially when smokers stop (30) and it is possible that this could be used as an another indication of abstinence in future trials.

Follow-up questionnaires will involve initial contact via e-mail with a link to an online survey. Embedded links will provide the option of responding to the main outcome measure assessed in that survey (at 4 
weeks, quit attempts; at 16 weeks, 12-week continuous abstinence; at 30 weeks, 26-week continuous abstinence) in a single click. Three attempts will be made one day apart for each follow-up. Follow-up will be attempted at all follow-up points regardless of success at a previous point. A mailing system will be used for the emails to enable assessment of whether the email was a) delivered versus hard bounce (i.e. permanent delivery problem) versus soft bounce (i.e. temporary delivery problem), and b) opened. Invitations and contacts will be structured according to evidence-based methods for maximisation of response rates $(31,32)$. All invitations will cite non-monetary incentives for responding (e.g. that the answer is important to advancing scientific knowledge of patterns of smoking behaviour and cessation and that a donation to a cancer research charity will be made for every person who responds) and will refer to university and charity funding of the trial. In order to boost the response rate for the final (30-week) followup, participants who provide their phone number in the baseline questionnaire and consent to receiving text messages will receive a text message asking them to reply "yes" or "no" in order to record their response to the primary outcome measure (i.e. 26-week continuous abstinence).

Debriefing will involve an email sent immediately after completion of the final follow-up. It will thank participants for their help with the study, remind them of the donation being made on their behalf and provide them with a contact email for further information.

\section{Withdrawal of participants}

Participants will be informed that they may withdraw from the study at any time without giving a reason. Unless they withdraw consent they will be followed up irrespective of smoking outcome or protocol violation. On the basis of the intention-to-treat principle, those who do not respond to endpoint follow-up attempts will be retained in the analyses and classified as continuing smokers. Participants who indicate that they no longer wish to take part in the study will be excluded from the analyses.

\section{Data analysis and management}

All variables will be collected primarily online and entered automatically into a Qualtrics database. From this database, a user-specified Excel file will be downloaded, and subjected to basic processing and re-coding, and integrated with responses provided by text messages. On completion, data will be analysed blind to intervention allocation by the trial statistician using $\mathrm{R}$.

Analysis of the primary outcome 
We will use log-binomial regression to calculate the relative risk (RR) and $95 \%$ confidence interval (Cl) of smoking cessation for at least 26 weeks, assessed 30 weeks after enrolment, in the intervention group compared with the comparator group.

\section{Analysis of the secondary outcomes}

We will use log-binomial regression to calculate the $\mathrm{RR}$ and $95 \% \mathrm{Cl}$ of (i) making at least one quit attempt, assessed 4 weeks after enrolment; (ii) smoking cessation for at least 12 weeks, assessed 16 weeks after enrolment; (iii) downloading or using the Smoke Free app, assessed 30 weeks after enrolment, in the intervention group compared with the comparator group.

In those who make at least one quit attempt in the 4 weeks following enrolment in the study, we will use log-binomial regression to calculate the $\mathrm{RR}$ and $95 \% \mathrm{Cl}$ of smoking cessation for at least 26 weeks, assessed 30 weeks after enrolment, in the intervention group compared with the comparator group.

\section{Moderation analyses}

For each primary and secondary endpoint, we will run a series of log-binomial regression models in which the two-way interactions between group (intervention, comparator) and sex (male, female), cigarette addiction (first cigarette within 5 minutes, 6-30 minutes, 31-60 minutes, >60 minutes after waking), age $(<35, \geq 35)$, education (post-16 qualifications: yes, no) financial situation (live comfortably, meet needs with a little left, just meet basic expenses, don't meet basic expenses) and previous experience with the app (previous experience: yes, no - inferred by matching email provided at baseline with mailing list) will be added.

\section{Sensitivity analyses}

We will repeat our primary and secondary analyses (i) restricting the intervention group to participants who take up the offer of full free access to the Smoke Free app, which will be verified by matching the email address used to login to the app to the one provided in the baseline questionnaire; and (ii) restricting both groups to participants who were successfully followed up. In order to aid interpretation of the strength of evidence for associations, we will calculate Bayes factors to differentiate between evidence for an effect, no effect and data insensitivity (33).

\section{Follow-on analysis}


In the event that our results are non-significant and insensitive (indicated by a Bayes factor between $1 / 3$ and $3(33)$ ), we will consult our trial steering committee to discuss the possibility of continuing to recruit until data are sensitive to reach a conclusion.

\section{Oversight committees}

A trial steering committee independent of the investigators, their employing organisations, funders and sponsors will provide overall supervision of the trial. They will monitor progress and advise on scientific credibility. A data monitoring committee will assess the progress of the trial annually or more often as required. This will ensure the accuracy of the data and maintain data quality. Data accuracy is just one component of data quality and refers to whether values stored for any variable are correct values. Data quality encompasses accuracy, absence of bias, and completeness of the information obtained from respondents (34).

\section{Dissemination policy}

Results will be disseminated to key stakeholders, and more broadly, in several ways. These include: openaccess peer-reviewed journal articles; presentation at key scientific meetings; feedback to trial participants; and press releases. Data and code will be made available on the Open Science Framework following publication. The criteria for authorship will be taken from the International Committee of Medical Journal Editors (35).

\section{Ethical approval}

Ethical approval for the trial will be obtained from the UCL Research Ethics Committee prior to commencement.

\section{Registration}

The study will be registered on the Open Science Framework and as an International Standard Randomised Controlled Trial.

\section{Discussion}


To date, no RCTs have been published comparing apps designed to provide ongoing support for quit attempts with unaided quitting. The Smoke Free app has been developed to be attractive and effective in promoting smoking cessation on the basis of behaviour change theory and empirical evidence $(21,24,25)$. In an exploratory RCT, the full version of the app was found to increase 3-month self-reported complete abstinence rates compared with a reduced version (21), demonstrating sufficiently promising efficacy to warrant further testing.

While the current evidence on the effectiveness of smartphone apps for smoking cessation is limited, a handful of studies have found higher success rates than would be expected from unaided cessation (12-19). Our trial will test the impact of a very brief intervention - the offer of a smartphone app - on quit attempts and cessation. This could provide leverage to include signposting to apps within routine primary care. Brief physician advice has been shown to increase the frequency of quit attempts, but offering assistance to smokers - for example, in the form of behavioural support or NRT - has a stronger effect (36). With apps offering a low-cost, low-resource, widely accessible option, they could provide a cost-effective method of supporting smokers to quit and reach a wider population of smokers who lack the time or inclination to try other behavioural or pharmacological cessation aids. Following the completion of our trial, it will be possible to estimate the cost-effectiveness of the intervention, and compare against other available smoking cessation interventions, using incremental cost-effectiveness tables (37). The widespread ownership of smartphones and the international setting of the trial mean that this type of smoking cessation intervention could potentially have an equity impact, narrowing socioeconomic inequalities in smoking prevalence and its associated health risks.

The study's results will need to be considered in the light of some caveats. First, because we cannot force people randomised to the experimental condition to use the app, the trial will test the effectiveness of an offer, rather than effectiveness of the app per se. As such, any effect we observe in our primary intentionto-treat analysis is likely to underestimate the true effectiveness of the app. We have planned a sensitivity analysis restricting our experimental group to those who took up the offer of the app to provide a comparative effect size.

Secondly, evaluating the effectiveness of smoking cessation apps versus unaided cessation in RCTs is complicated by the fact that apps are widely available and participants who are randomised to the unaided quitting condition are likely to drop out of the study or use one of the many freely available apps. Loss to follow-up is a key issue for all smoking cessation trials. However, with sufficient resources, high follow-up 
rates can be obtained in this type of trial $(13,38)$. We will use email and text message reminders, simplified options for reporting the key outcomes, and charitable donations as an incentive to ensure that retention remains as high as possible.

Thirdly, in considering participants lost to follow-up as having continued or resumed smoking (36), an intention-to-treat approach may bias effect sizes downwards if loss to follow-up occurs for reasons other than relapse to smoking (39), or upwards if the intervention condition leads to higher follow-up rates than the control condition. Only including participants who complete the follow-up can overcome bias caused by differential follow-up between groups, but may overestimate success rates in both groups if participants who fail to quit refuse to participate in follow-up assessments. In practice, 'missing equals smoking' and 'follow-up only' approaches tend to produce very similar odds ratios in smoking cessation RCTs (40), though the percentage point difference between conditions varies considerably. To address biases arising from loss to follow-up, we will use both approaches to analyse the trial results. It is likely that the true percentage point difference and odds ratios will lie somewhere between the estimates provided by these two methods.

Finally, biochemical verification of abstinence is recommended in smoking cessation trials because of psychological pressure to claim abstinence (36). However, this is highly resource intensive - particularly given the international setting for the trial - and may undermine generalisability to smokers who would use an app but not a more intensive interaction. Remote carbon monoxide testing has been shown to yield low response rates in the context of a smoking cessation app trial with no direct contact with researchers (41). With the low-key nature of the intervention (a brief email offer of free use of the app) offering little more in the way of encouragement to quit than the control condition, there should be little difference in the social pressure to claim abstinence between conditions, so the use of self-report should not bias the estimated effect size. In an effort to verify this, resting heart rate measurements will be collected at baseline and final follow-up from participants who report owning a heart monitoring device (e.g. FitBit).

To summarise, if the Smoke Free smartphone app is found to be effective in promoting smoking cessation, it could provide an affordable and accessible option for cessation support for smokers around the world. 


\section{Acknowledgments}

\section{Author contributions}

SEJ drafted the manuscript. All authors provided critical revisions and approved the final manuscript.

\section{Declarations of interest}

DC is originator of the Smoke Free app and derives income from it. RW has undertaken research and consultancy for and receives travel funds and hospitality from manufacturers of smoking cessation medications (Pfizer, GlaxoSmithKline and Johnson and Johnson). JB has received unrestricted research funding from Pfizer, who manufacture smoking cessation medications. RW and JB are both unpaid members of the scientific steering group of the Smoke Free mobile application.

\section{Funding}

Cancer Research UK fund SJ, OP \& JB's salary (C1417/A22962). SJ's salary was also supported by the ESRC (ES/R005990/1). The funders had no final role in the study design; in the writing of the report; or in the decision to submit the paper for publication. All researchers listed as authors are independent from the funders and all final decisions about the research were taken by the investigators and were unrestricted. 


\section{References}

1. World Health Organization. Global status report on noncommunicable diseases 2014. [Internet]. 2014 [cited 2018 Aug 8]. Available from: http://www.who.int/nmh/publications/ncd-status-report2014/en/

2. Office of the Surgeon General (US), Office on Smoking and Health (US). The Health Consequences of Smoking: A Report of the Surgeon General [Internet]. Atlanta (GA): Centers for Disease Control and Prevention (US); 2004 [cited 2018 Aug 7]. (Reports of the Surgeon General). Available from: http://www.ncbi.nlm.nih.gov/books/NBK44695/

3. Hyland A, Borland R, Li Q, Yong H-H, McNeill A, Fong GT, et al. Individual-level predictors of cessation behaviours among participants in the International Tobacco Control (ITC) Four Country Survey. Tob Control. 2006 Jun 1;15(suppl 3):iii83-94.

4. Centers for Disease Control and Prevention (CDC). Cigarette smoking among adults--United States, 2007. MMWR Morb Mortal Wkly Rep. 2008 Nov 14;57(45):1221-6.

5. Panel TU and DG. Treating Tobacco Use and Dependence: 2008 Update. US Department of Health and Human Services; 2008.

6. West R, Shahab L. Smoking cessation interventions. In: Evidence-based Public Health: Effectiveness and Efficiency. Oxford: Oxford University Press; 2010. p. 215-232.

7. Pulverman R, Yellowlees PM. Smart devices and a future of hybrid tobacco cessation programs. Telemed J E-Health Off J Am Telemed Assoc. 2014 Mar;20(3):241-5.

8. Abroms LC, Padmanabhan N, Thaweethai L, Phillips T. iPhone apps for smoking cessation: a content analysis. Am J Prev Med. 2011 Mar;40(3):279-85.

9. Abroms LC, Lee Westmaas J, Bontemps-Jones J, Ramani R, Mellerson J. A content analysis of popular smartphone apps for smoking cessation. Am J Prev Med. 2013 Dec;45(6):732-6.

10. Zenith. Smartphone penetration to reach $66 \%$ in 2018 [Internet]. Zenith. 2017 [cited 2018 Aug 7]. Available from: https://www.zenithmedia.com/smartphone-penetration-reach-66-2018/

11. Annual number of global mobile app downloads 2017-2022 [Internet]. Statista. 2018 [cited 2018 Aug 7]. Available from: https://www.statista.com/statistics/271644/worldwide-free-and-paid-mobile-appstore-downloads/

12. Taylor GMJ, Dalili MN, Semwal M, Civljak M, Sheikh A, Car J. Internet-based interventions for smoking cessation. Cochrane Database Syst Rev. 2017 04;9:CD007078.

13. Bricker JB, Mull K, Kientz JA, Vilardaga RM, Mercer LD, Akioka K, et al. Randomized, Controlled Pilot Trial of a Smartphone App for Smoking Cessation Using Acceptance and Commitment Therapy. Drug Alcohol Depend. 2014 Oct 1;143:87-94. 
14. Buller DB, Borland R, Bettinghaus EP, Shane JH, Zimmerman DE. Randomized trial of a smartphone mobile application compared to text messaging to support smoking cessation. Telemed J E-Health Off J Am Telemed Assoc. 2014 Mar;20(3):206-14.

15. Garrison KA, Pal P, O'Malley SS, Pittman BP, Gueorguieva R, Rojiani R, et al. Craving to Quit: A Randomized Controlled Trial of Smartphone App-Based Mindfulness Training for Smoking Cessation. Nicotine Tob Res [Internet]. [cited 2018 Aug 23]; Available from: https://academic.oup.com/ntr/advance-article/doi/10.1093/ntr/nty126/5039853

16. Bricker JB, Copeland W, Mull KE, Zeng EY, Watson NL, Akioka KJ, et al. Single-arm trial of the second version of an acceptance \& commitment therapy smartphone application for smoking cessation. Drug Alcohol Depend. 2017 01;170:37-42.

17. lacoviello BM, Steinerman JR, Klein DB, Silver TL, Berger AG, Luo SX, et al. Clickotine, A Personalized Smartphone App for Smoking Cessation: Initial Evaluation. JMIR MHealth UHealth. 2017 Apr 25;5(4):e56.

18. Ubhi HK, Michie S, Kotz D, Wong WC, West R. A mobile app to aid smoking cessation: preliminary evaluation of SmokeFree28. J Med Internet Res. 2015 Jan 16;17(1):e17.

19. Whittaker R, McRobbie H, Bullen C, Rodgers A, Gu Y. Mobile phone-based interventions for smoking cessation. Cochrane Database Syst Rev [Internet]. 2016 [cited 2018 Aug 23];(4). Available from: https://www.cochranelibrary.com/cdsr/doi/10.1002/14651858.CD006611.pub4/abstract

20. BinDhim NF, McGeechan K, Trevena L. Smartphone Smoking Cessation Application (SSC App) trial: a multicountry double-blind automated randomised controlled trial of a smoking cessation decision-aid 'app.' BMJ Open. 2018 Jan 1;8(1):e017105.

21. Crane D, Ubhi HK, Brown J, West R. Relative effectiveness of a full versus reduced version of the 'Smoke Free' mobile application for smoking cessation: a randomised controlled trial. F1000Research. 2018 Sep 21;7:1524.

22. Ferguson SG, Brown J, Frandsen M, West R. Associations between use of pharmacological aids in a smoking cessation attempt and subsequent quitting activity: a population study. Addict Abingdon Engl. 2015 Mar;110(3):513-8.

23. West R, Evins AE, Benowitz NL, Russ C, McRae $T$, Lawrence $D$, et al. Factors associated with the efficacy of smoking cessation treatments and predictors of smoking abstinence in EAGLES. Addiction. 2018 Aug 1;113(8):1507-16.

24. West R, Evans A, Michie S. Behavior change techniques used in group-based behavioral support by the English stop-smoking services and preliminary assessment of association with short-term quit outcomes. Nicotine Tob Res Off J Soc Res Nicotine Tob. 2011 Dec;13(12):1316-20.

25. Ubhi HK, Michie S, Kotz D, van Schayck OCP, Selladurai A, West R. Characterising smoking cessation smartphone applications in terms of behaviour change techniques, engagement and ease-of-use features. Transl Behav Med. 2016;6(3):410-7. 
26. Michie S, Hyder N, Walia A, West R. Development of a taxonomy of behaviour change techniques used in individual behavioural support for smoking cessation. Addict Behav. 2011 Apr;36(4):315-9.

27. West R, Hajek P, Stead L, Stapleton J. Outcome criteria in smoking cessation trials: proposal for a common standard. Addict Abingdon Engl. 2005 Mar;100(3):299-303.

28. Kotz D, Brown J, West R. Predictive validity of the Motivation To Stop Scale (MTSS): A single-item measure of motivation to stop smoking. Drug Alcohol Depend. 2013 Feb 1;128(1):15-9.

29. Williams VF, Smith AA, Villanti AC, Rath JM, Hair EC, Cantrell J, et al. Validity of a Subjective Financial Situation Measure to Assess Socioeconomic Status in US Young Adults. J Public Health Manag Pract. 2017 Oct;23(5):487.

30. Minami J, Ishimitsu T, Matsuoka H. Effects of smoking cessation on blood pressure and heart rate variability in habitual smokers. Hypertension. 1999;33(1):586-590.

31. Free C, Knight R, Robertson S, Whittaker R, Edwards P, Zhou W, et al. Smoking cessation support delivered via mobile phone text messaging (txt2stop): a single-blind, randomised trial. The Lancet. 2011 Jul 2;378(9785):49-55.

32. Edwards $\mathrm{P}$, Roberts I, Clarke $\mathrm{M}$, DiGuiseppi $\mathrm{C}$, Pratap $\mathrm{S}$, Wentz $\mathrm{R}$, et al. Increasing response rates to postal questionnaires: systematic review. BMJ. 2002 May 18;324(7347):1183.

33. Beard E, Dienes Z, Muirhead C, West R. Using Bayes factors for testing hypotheses about intervention effectiveness in addictions research. Addict Abingdon Engl. 2016;111(12):2230-47.

34. Bowling A. Mode of questionnaire administration can have serious effects on data quality. J Public Health. 2005 Sep 1;27(3):281-91.

35. ICMJE | Recommendations | Defining the Role of Authors and Contributors [Internet]. [cited 2018 Dec 19]. Available from: http://www.icmje.org/recommendations/browse/roles-andresponsibilities/defining-the-role-of-authors-and-contributors.html

36. Aveyard P, Begh R, Parsons A, West R. Brief opportunistic smoking cessation interventions: a systematic review and meta-analysis to compare advice to quit and offer of assistance. Addiction. 2012;107(6):1066-73.

37. Stapleton JA, West R. A direct method and ICER tables for the estimation of the cost-effectiveness of smoking cessation interventions in general populations: application to a new cytisine trial and other examples. Nicotine Tob Res Off J Soc Res Nicotine Tob. 2012 Apr;14(4):463-71.

38. Brown J, Michie S, Geraghty AW, Yardley L, Gardner B, Shahab L, et al. Internet-based intervention for smoking cessation (StopAdvisor) in people with low and high socioeconomic status: a randomised controlled trial. Lancet Respir Med. 2014 Dec 1;2(12):997-1006.

39. Barnes SA, Larsen MD, Schroeder D, Hanson A, Decker PA. Missing data assumptions and methods in a smoking cessation study. Addict Abingdon Engl. 2010 Mar;105(3):431-7. 
40. Blankers $\mathrm{M}$, Smit ES, van der Pol $\mathrm{P}$, de Vries $\mathrm{H}$, Hoving $\mathrm{C}$, van Laar $\mathrm{M}$. The Missing=Smoking Assumption: A Fallacy in Internet-Based Smoking Cessation Trials? Nicotine Tob Res Off J Soc Res Nicotine Tob. 2016 Jan;18(1):25-33.

41. Herbec A, Brown J, Shahab L, West R. Lessons learned from unsuccessful use of personal carbon monoxide monitors to remotely assess abstinence in a pragmatic trial of a smartphone stop smoking app - A secondary analysis. Addict Behav Rep [Internet]. 2018 Jul 23 [cited 2019 Jan 9]; Available from: http://www.sciencedirect.com/science/article/pii/S2352853218300634 


\section{Tables}

Table 1. Participant eligibility criteria

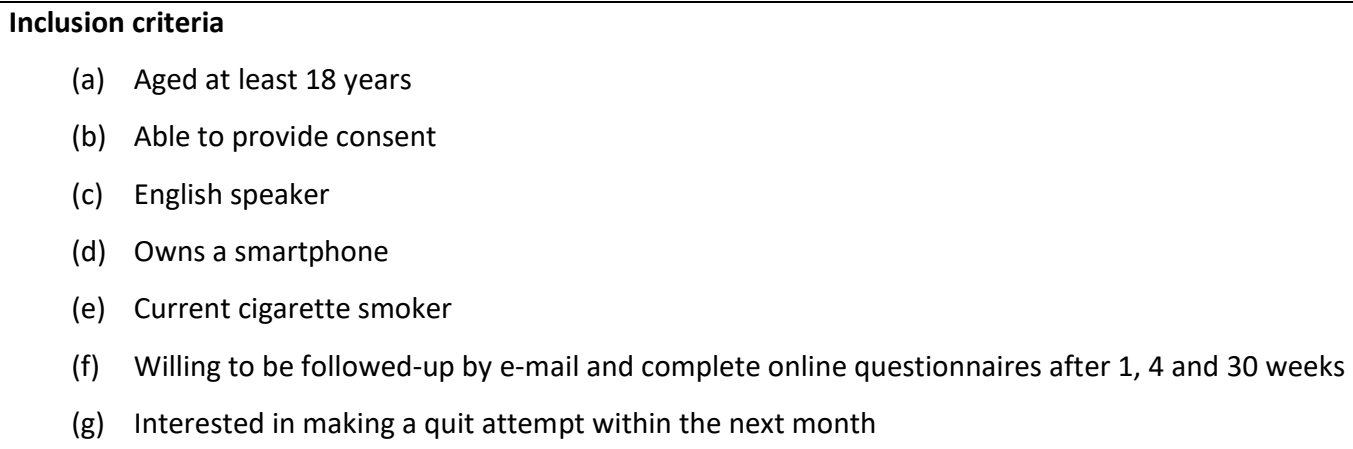

(a) Aged at least 18 years

(b) Able to provide consent

(c) English speaker

(d) Owns a smartphone

(e) Current cigarette smoker

(f) Willing to be followed-up by e-mail and complete online questionnaires after 1, 4 and 30 weeks

(g) Interested in making a quit attempt within the next month

Exclusion criteria

(a) Used the Smoke Free app in the past 6 months 
Table 2. Behaviour change techniques (BCTs) included in the Smoke Free app

\begin{tabular}{|c|c|c|}
\hline BCT Code and Label & $\begin{array}{l}\text { BCT } \\
\text { Present?* }\end{array}$ & $\begin{array}{l}\text { Instantiation in the Smoke Free } \\
\text { app }\end{array}$ \\
\hline \multicolumn{3}{|l|}{ Specific focus on behaviour (B) and addressing motivation (M) } \\
\hline BM1. Provide information on consequences of smoking and smoking cessation & $\checkmark$ & Chatbot \\
\hline BM2. Boost motivation and self-efficacy & $\checkmark$ & Badges, Chatbot \\
\hline BM3. Provide feedback on current behaviour & $\checkmark$ & Dashboard \\
\hline BM4. Provide rewards contingent on successfully stopping smoking & $\checkmark$ & Badges, Chatbot \\
\hline \multicolumn{3}{|l|}{ BM5. Provide normative information about others' behaviour and experiences } \\
\hline BM6. Prompt commitment from the client here and then & $\checkmark$ & Registration; Chatbot \\
\hline BM7. Provide rewards contingent on effort or progress & $\checkmark$ & Badges, Chatbot \\
\hline BM8. Strengthen ex-smoker identity & $\checkmark$ & Chatbot \\
\hline BM9. Identify reasons for wanting and not wanting to stop smoking & $\checkmark$ & Chatbot \\
\hline BM10. Explain the importance of abrupt cessation & $\checkmark$ & Chatbot \\
\hline \multicolumn{3}{|l|}{ BM11. Measure expired-air carbon monoxide (CO) concentration } \\
\hline \multicolumn{3}{|l|}{ Specific focus on behaviour (B) and maximising self-regulatory capacity/skills (S) } \\
\hline BS1. Facilitate barrier identification and problem solving & $\checkmark$ & Chatbot \\
\hline BS2. Facilitate relapse prevention and coping & $\checkmark$ & Chatbot \\
\hline BS3. Facilitate action planning/develop treatment plan & $\checkmark$ & Cravings; Chatbot \\
\hline BS4. Facilitate goal setting & $\checkmark$ & Registration; Chatbot \\
\hline BS5. Prompt review of goals & $\checkmark$ & Diary; Badges, Chatbot \\
\hline BS6. Prompt self-recording & $\checkmark$ & Diary; Chatbot \\
\hline BS7. Advise on changing routine & $\checkmark$ & Chatbot \\
\hline BS8. Advise on environmental restructuring & $\checkmark$ & Chatbot \\
\hline \multicolumn{3}{|l|}{ BS9. Set graded tasks } \\
\hline BS10. Advise on conserving mental resources & $\checkmark$ & Chatbot \\
\hline BS11. Advise on avoiding social cues for smoking & $\checkmark$ & Chatbot \\
\hline \multicolumn{3}{|l|}{ Promote adjuvant activities (A) } \\
\hline A1. Advise on stop-smoking medication & $\checkmark$ & Chatbot \\
\hline A2. Advise on/facilitate use of social support & $\checkmark$ & Chatbot \\
\hline \multicolumn{2}{|l|}{ A3. Adopt appropriate local procedures to enable clients to obtain free } & \\
\hline \multicolumn{3}{|l|}{ A4. Ask about experiences of stop smoking medication that the smoker is using } \\
\hline \multicolumn{3}{|l|}{ A5. Give options for additional and later support } \\
\hline \multicolumn{3}{|l|}{ General aspects of the interaction $(R)$ focusing on the delivery of the intervention $(D)$} \\
\hline RD1. Tailor interactions appropriately & $\checkmark$ & Chatbot \\
\hline
\end{tabular}




\begin{tabular}{|c|c|c|}
\hline RD2. Emphasise choice & $\checkmark$ & Chatbot \\
\hline \multicolumn{3}{|c|}{ General aspects of the interaction (R) focusing on information gathering (I) } \\
\hline RI1. Assess current and past smoking behaviour & $\checkmark$ & Registration, Chatbot \\
\hline \multicolumn{3}{|l|}{ RI2. Assess current readiness and ability to quit } \\
\hline \multicolumn{3}{|l|}{ RI3. Assess past history of quit attempts } \\
\hline \multicolumn{3}{|l|}{ RI4. Assess withdrawal symptoms } \\
\hline \multicolumn{3}{|c|}{ General aspects of the interaction (R) focusing on general communication (C) } \\
\hline RC1. Build general rapport & $\checkmark$ & Throughout \\
\hline \multicolumn{3}{|l|}{ RC2. Elicit and answer questions } \\
\hline \multicolumn{3}{|l|}{ RC3. Explain the purpose of CO monitoring } \\
\hline RC4. Explain expectations regarding treatment programme & $\checkmark$ & Chatbot \\
\hline RC5. Offer/direct towards appropriate written materials & $\checkmark$ & Chatbot \\
\hline RC6. Provide information on withdrawal symptoms & $\checkmark$ & Chatbot \\
\hline \multicolumn{3}{|l|}{ RC7. Use reflective listening } \\
\hline \multicolumn{3}{|l|}{ RC8. Elicit client views } \\
\hline \multicolumn{3}{|l|}{ RC9. Summarise information/confirm client decisions } \\
\hline RC10. Provide reassurance & $\checkmark$ & Chatbot \\
\hline
\end{tabular}

Note. * BCT presence is indicated by a tick. 
Table 3. Schedule of enrolment and follow-up assessments

\begin{tabular}{|c|c|c|c|c|}
\hline \multirow[b]{2}{*}{ Assessment } & \multicolumn{4}{|c|}{ Time point } \\
\hline & Baseline & 4 weeks & 16 weeks & 30 weeks \\
\hline Initial approach & $x$ & & & \\
\hline Informed consent & $x$ & & & \\
\hline Eligibility screen & $x$ & & & \\
\hline Randomisation & $x$ & & & \\
\hline Intervention/comparator initiation & $x$ & & & \\
\hline Sociodemographic characteristics ${ }^{1}$ & $x$ & & & \\
\hline Cigarette consumption and level of addiction & $x$ & $x$ & $x$ & $\mathrm{x}$ \\
\hline Quit history and past use of cessation support & $x$ & & & \\
\hline Current use of cessation support & $x$ & $x$ & $\mathrm{x}$ & $x$ \\
\hline Quit attempts & & $x$ & & \\
\hline Smoking cessation & & $x$ & $x$ & $x$ \\
\hline App use & & & & $x$ \\
\hline Resting heart rate ${ }^{2}$ & $x$ & & & $x$ \\
\hline Debriefing & & & & $x$ \\
\hline
\end{tabular}

${ }^{1}$ Sociodemographic characteristics include: age, sex, education, subjective financial status, country of residence and first language.

${ }^{2}$ Current resting heart rate will be self-reported by participants who have a heart rate monitoring device (e.g. FitBit). 